IRA-International Journal of Applied Sciences ISSN 2455-4499; Vol.07, Issue 03 (2017)

Institute of Research Advances

Pg. no. 111-117

https://research-advances.org/index.php/IRAJAS

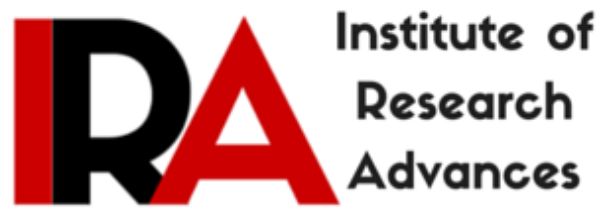

\title{
Evaluation of Carbon Nanotubes Making Techniques
}

Mansoureh Pourjafar

Higher Educational Complex of Saravan, Saravan, Sistan and Baluchestan, Iran.

Type of Review: Peer Reviewed.

DOI: http://dx.doi.org/10.21013/jas.v7.n3.p2

How to cite this paper:
Pourjafar, M. (2017). Evaluation of Carbon Nanotubes Making Techniques. IRA-International Journal of Applied Sciences (ISSN 2455-4499), 7(3), 111-117. doi:http://dx.doi.org/10.21013/jas.v7.n3.p2

(C) Institute of Research Advances

\section{(cc) BY-NC}

This work is licensed under a Creative Commons Attribution-Non Commercial 4.0 International License subject to proper citation to the publication source of the work.

Disclaimer: The scholarly papers as reviewed and published by the Institute of Research Advances (IRA) are the views and opinions of their respective authors and are not the views or opinions of the IRA. The IRA disclaims of any harm or loss caused due to the published content to any party. 
After the initial discovery of Carbon Nanotubes in the Grime by electrical discharge, various methods have been developed to produce Carbon Nanotubes. In this study, four more conventional methods for producing Carbon Nanotubes have been evaluated. These include: Electrical arc discharge, Laser ablation, Sedimentation by chemical vapor and HIPco. The last method mentioned is highly regarded in the last few years and it have been produced the subject of many investigations in the field of Carbon Nanotubes. The results of this research show that HIPco production method has ability to supply mass production of Carbon Nanotubes in the future.

Keywords: Nanotechnology, Carbon Nanotubes (CNT), carbon nanotube manufacturing techniques.

\section{Introduction}

Until the before since 1985 the only known examples of carbon were limited to graphite and diamond. In 1985, the third type of carbon was introduced that has major difference whit the known examples [4].This type of carbon called fullerenes were not found in nature and was produced by a laser power. Fullerenes is formed of 60 carbon atoms in a closed spherical structure (Fig. 1). Kratschmer In 1990 found that using electric arc between graphite electrodes, are produced carbon Grime containing $C_{60}$ and other fullerenes [4]. He obtained ability to produce fullerenes in small quantities $(\mathrm{g})$ in the laboratory, using the device relatively easy that raised intensity level of research activities related to these molecules and cause creation a renaissance in the study carbon.

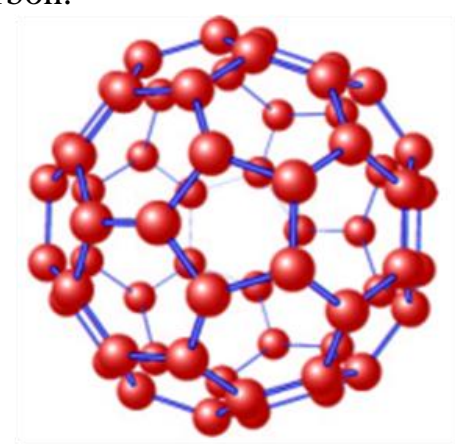

Figure 1. Structure of fullerenes that are shaped of 60 carbon atoms and in a spherical surface.

In 1991 Lijima when making fullerenes observed that the direct current electric arc, tubes Nano-graphite precipitate on the negative electrode [8]. The nanotubes were cylinders of graphite with a closed end that have been formed form of single-layer and multi-layers (Fig. 2). After this discovery, research on development, identifying characteristics and applications of nanotubes spread explosively.

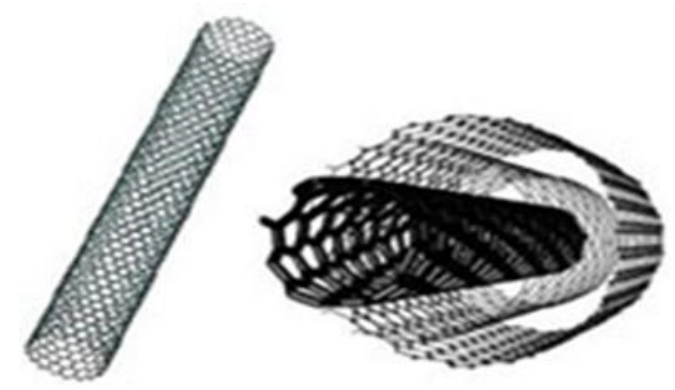

Figure 2.Single-layer and multi-layer nanotubes.

Carbon Nanotubes (CNT) could be metallic or semi-metal (semiconductor), so provides the possibility of connecting the semiconductor - semiconductor and semiconductor-conductive for use in components [5]. 
High tensile strength and Young's modulus, and other mechanical properties, take advantage of composites very resistant Creates building industry. Many researchers are investigating the potential of nanotubes in a wide range of applications. Some of these applications include: Nano electronics, sensors, Propagation field, displays, hydrogen storage, batteries, polymer composites, wear bullet-proof, reinforced materials, Nano-scale reactors and electrodes [10].

Nanotubes are one of the strongest material and best fibers that is made of graphite structure Theoretical studies has proposed, the Young's modulus in the range of 1-5 tpa for nanotubes (Modulus of elasticity diamond is 1.2 tpa). The real strength of multi-walled nanotubes, are affected the slip graphene layer on each other.

The most interesting properties of nanotubes are related to its electronic band structure. Initial calculations showed that the nanotube depending on the chirality vector $(n, m)$ and diameters can be conductive or semi conductive. Type armchair always is conductive while zigzag type can be conductive or semi-conductive (Figure 3)

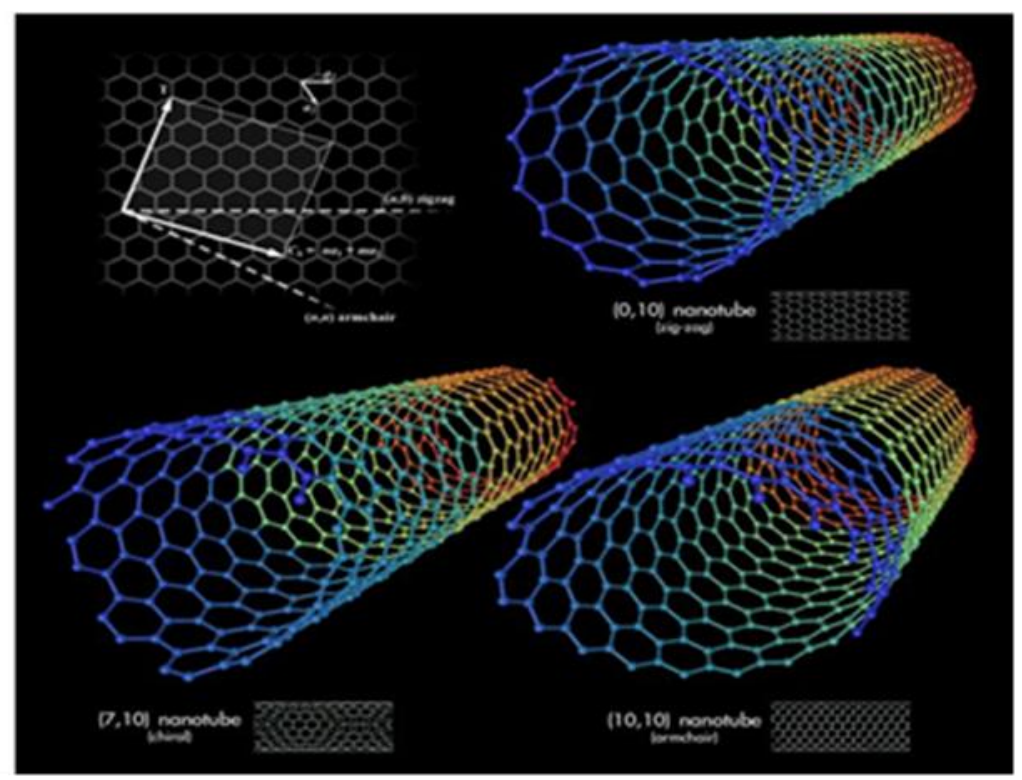

Figure 3. Type of nanotubes, zigzag, chiral and chairs

\section{Structural defects in nanotubes}

Several types of defects in Carbon Nanotubes are affected structures and connections that can be cited the following examples: impurities localized in the network, the adsorption of molecules, defects pentagon and heptagon, carbonization (becoming amorphous carbon).

1. Impurities localized in the network: Impurities usually are in the form of atoms that are combined in the existing carbon nanotube network. And they take instead of the carbon atoms in the network. Atoms such as boron and nitrogen are atoms of the species. However, localized atoms in the unit cell will change the shape of Carbon Nanotubes and whereby will change electrical properties and bonding (connection).

2. The adsorption of molecules: the molecules that are absorbed by the nanotubes surfaces, changed the unit cell in CNT, and therefore change electrical properties. Among them, is investigated No2 and $\mathrm{NH} 3$ adsorption because it is more common [1]. 
3. Defects pentagon and heptagon: defects pentagon and heptagon are differ from other defects. These defects are structural defects that have been observed in Carbon Nanotubes. Rings of pentagons in reverse are formed, right in front of seven hexagonal rings. These defects cause CNT to be curved Heptagon. So can by distribution a pair of defects in the nanotubes, it created a spiral shape. In most such structures, can be seen in vitro observations nanotubes, (Figure 4).
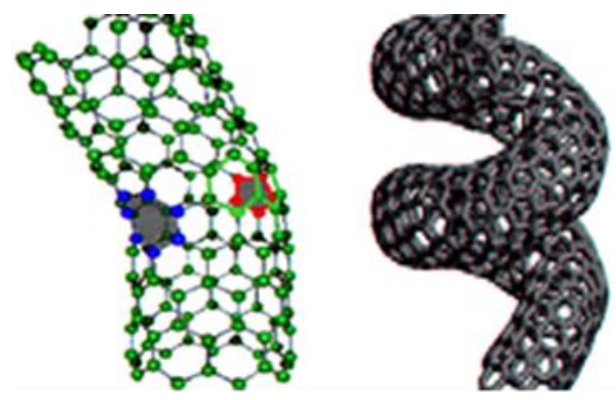

Figure 4. Defects caused by torsion pentagon and heptagon

Pentagon and heptagon defects have been observed in Carbon Nanotubes. Such a defect appears then that a nanotube Arm Chair placed under tension in line with its axis. This defect cause the tensile strength of the nanotube and with the loss of traction force, refers to a previous state. This mode can be seen in Figure 5.

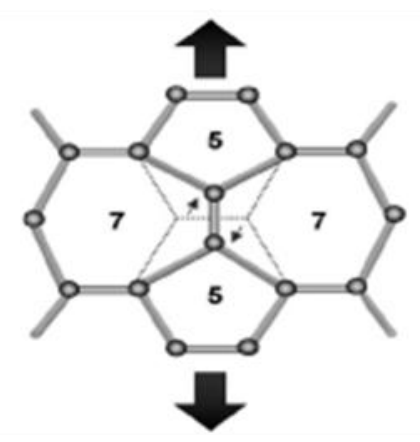

Figure 5.Structure of the pentagon and heptagon in effect tension on the nanotube surface.

4. Carbonization (becoming amorphous carbon): carbonization nanotubes that is known as one of defects nanotubes, occurs when that carbon atoms not perch within regular network. The emergence of this defect refers to methods of making nanotube [2].

\section{Methods of making Carbon Nanotubes}

After the initial discovery of Carbon Nanotubes in the Grimeby electrical discharge, various methods have been developed to produce Carbon Nanotubes. In this study, four more conventional methods for producing Carbon Nanotubes have been evaluated. These include: Electrical arc discharge, Laser ablation, Sedimentation by chemical vapor and HIPco.

\section{Electrical arc discharge:}

The arc discharge method, there is a simple method for carbon vapor in a plasma that finally structure to produce high-quality nanotubes turns. In figures 6 and 7 is described, an outline of the device producing Carbon Nanotubes in the arc discharge method. The anode and cathode are carbon rods with a diameter of 5 to 20 millimeter, since thereafter anodes resurrection and began evaporation end, is changed the distance between the anode and the cathode, this machine settings so that all nanotubes production time, keeps a constant distance. 


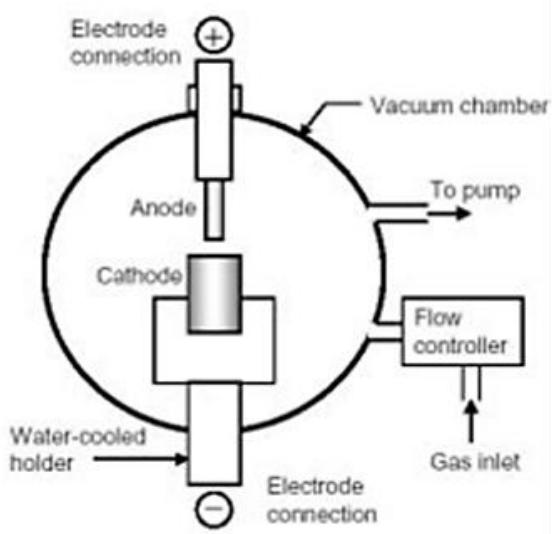

Figure 6.This figure is the general idea of an electrical arc discharge machine. The anode and cathode, that both of them are made of carbonate first, are together at the right distance. When a large flow from the anode is passing to the cathode a discharge arc will be created. Inside this arc discharge a carbonic plasma is formed and Carbon Nanotubes sediment the on the cathode.

The potential difference between the cathode and anode is about 20 to $25 \mathrm{~V} \mathrm{dc}$, which is caused by the flow of about 50 to 120 ampr. During production operations, must flow the helium gas whit velocity at 5 to $15(\mathrm{ml}$ per second) chamber devices and also as the chamber pressure is 500 Torr times.( 1 Torr $=133.32$ Pascals)

Carbon Nanotubes are produced bundles in the cathode internal temperature is higher than other parts.

Bundles nanotubes place an aligned along the stream. Efficiency, quality and type of nanotube is produced, greatly depends on the conditions of nanotube growth so far has been the highest yields about $20 \%$ graphite evaporated, that this is only achieved in the center of cathode.

Also can created use other catalysts on graphite rod, possible variations in specific nanotubes and produce single-walled Carbon Nanotubes, multi-walled Carbon Nanotubes, single-walled nanotube strands of single-walled nanotubes. Other carbon particles are sedimentation in the walls of the chamber and the surrounding nanotubes [7].

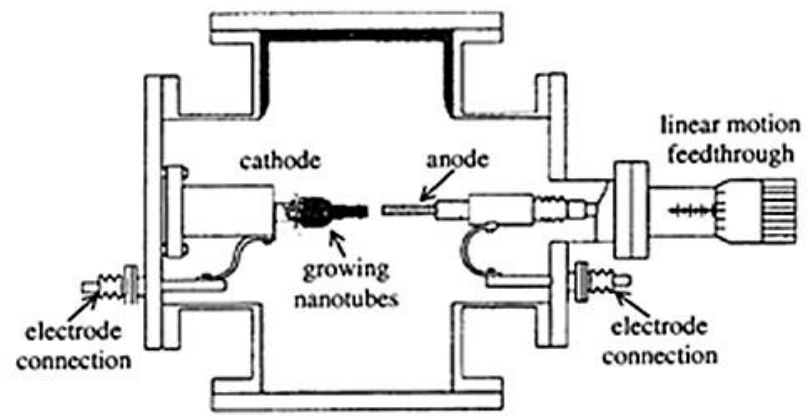

Figure 7.Schematic an electrical arc discharge device for the production of Carbon Nanotubes.

\section{Laser abrasion}

A very effective way to produce single-walled Carbon Nanotubes fields, is using powerful lasers to vaporize a sample of the graphite. The graphite samples are mixed with metals such as cobalt and nickel. The working principles of the system are shown in Figure 2. The purpose of the graphite placed in the furnaces and collisions the laser beam with that. Just the front of the laser and outside furnace is placed a water-cooled. The cooler has a copper disc to provide cooling as a collector during the process, causing sedimentation of the Carbon Nanotubes. 
A constant flow of argon gas at a temperature of about $1200^{\circ} \mathrm{C}$, from the laser to the collector of copper, makes the flow of carbon-evaporated moves into the collector and collided to cooled copper, and will sit Single-wall nanotubes on the collector.

SWCNT fibers produced generally have a diameter in the range of 10 to $20 \mathrm{~nm}$ and a length of up to 100 micrometers. Some specific nanotubes were observed with a diameter of about 1 to $3 \mathrm{~nm}$.

The range diameter of the nanotube is generally very low amplitude. Furnace temperature and other parameters affect on the diameter of the nanotubes are produced.

\section{Deposition by Chemical vapor}

Deposition by chemical vapor that is called for short CVD, is a generic term for several methods.

In all these methods, there is the carbon evaporation process and the use of catalyst particles. Carbon vapor produced by hydrocarbons that the hydrocarbons is exasperated in a "two-area flow reactor". In this reactor, there is a weak stream of $\mathrm{H} 2$ uniformly, which in some ways is mixed with a carrier gas (Figure $8)$.

Gas flow, carbon molecules evaporated transmit to an area with higher temperature (about 900 to $1100^{\circ}$ C), with the onset of the reaction of carbon vapor and catalyst particles, begins creation Carbon Nanotubes [9]. After the formation of nanotubes, can be separated catalyst particles into two types of nanotubes. The particles well remain in the hydrocarbon as well by techniques such as electron beam and lithography are precipitated on the surface.

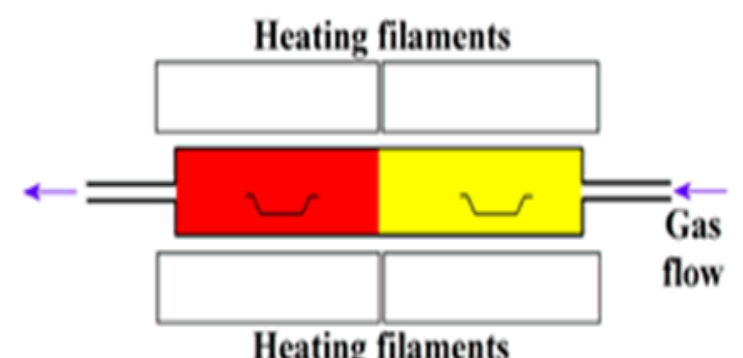

Figure 8.This figure is a plan of a two-area flow reactor. Hydrocarbons placed in area that evaporates with temperature of that area (yellow). A constant flow of hydrogen gas, transmissions carbon molecules in to the red area with a temperature of 900 to $1100{ }^{\circ}$.In this area with the reaction is carried out with catalyst particles, multi-walled Carbon Nanotubes are created [3].

\section{High pressure carbon monoxide process}

High pressure carbon monoxide process principles (HiPco), is inserted on the principles similar to the principles-based CVD. In this process, is provided steam carbon atoms by carbon monoxide gas .This gas pump steadily to chamber pressure of about 30- 50 atmospheres. The enclosure it works at a high temperature in the range of $900-1100^{\circ} \mathrm{C}$ and contains gas industry to take adequate amount of catalyst particles. Carbon monoxide mixed with the gas industry and catalyst particles with constant reaction parameters cause the production of Carbon Nanotubes.

The temperature and pressure required in HiPco to an extent that does not need to design special devices and they can be created by the same generic devices available in the industry. For this reason, this method is known less costly and faster than other methods in the production of SWCNT. In laboratory Rice, the current research on the process parameters are optimized so that has made it possible for produce 250 grams SWCNT in less than a week.

\section{Conclusion}

1. Electrical arc discharge method is a simple and inexpensive method for manufacturing nanotubes near perfect. One of the most disadvantages of this method compared to other methods is the lack of control on 
the surface formation of nanotubes. In this way, there is no way to produce nanotubes product in a favorable position in the machine. So shaped nanotubes may be mixed with several carbon particles these events, unavoidable refine of nanotubes.

2. Laser ablation method, is probably the best method for making SWCNT without fault. This method, unlike the cheap electrical arc discharge method, a method is relatively expensive and the most cost is related to provide powerful lasers.

3. The method of settling of catalyst particles is advantageous compared to the way in which the catalysts remain in hydrocarbons. The exact position grow Carbon Nanotubes can be determined in the CVD method can by controlling the electric field, to limit grow the nanotubes in place. Also by this field can be developed and organized groups of nanotubes in parallel and prevented intertwined nanotubes.

4. To increase the diameter and wall nanotubes can change factors such as size and type of catalyst particles and input gas, or by controlling to achieved the desired types of nanotubes. Also a type nanotube depends on the reactor temperature and gas flow input. Despite the above possibilities CVD, this method is used to produce large volumes of multi-walled nanotubes with high defect density.

5. Although there is no way to control the deposition of the CVD method, the only advantage HiPco process is efficiency and high speed production. Patented this method is in the laboratory of Rice and probably the way will become to SWCNT mass production source for the research and industry in the future. This method is highly regarded in recent years and has been the subject of many investigations the sphere of production of Carbon Nanotubes. The results of this research have shown that HIPco production method has its ability to mass production source of Carbon Nanotubes in the future.

\section{Reference}

1. Dohn,S., 2003."Investigation of mechano-electrical properties of multi-walled Carbon Nanotubes" at the Micro- and Nanotechnology Research Center, Technical University of Denmark.

2. Galanov,B.A. Galanov, S.B. Gogotsi, Y.J., 2002." Stress-strain state of multiwall carbon nanotube under internal pressure " Nanoparticle Res.

3. Lundey, Anders Mathias Jauhoz,Antti-Pekka., 2001"Coulomb Drag in Multiwall Carbon Nanotubes",Niels Bohr Institute, Ârsted Laboratory, University of Copenhagen, Copenhagen, Denmark.

4. Meyyappan,M. SrivastavaD., 2003."Carbon Nanotubes",chapter 18 of hand book of CRC Press LLC.

5. Narayan, R.Aluru, Jean-Pierre Leburton.,2003."Modeling Electronics at the Nanoscale", chapter 11 of hand book of CRC Press LLC.

6. pkollar,George, L. sprlnger, s., 2003. "Mechanics of Composite Structures" CAMBRIDGE university .

7. Popov, V. N. VavDorenV. E., and Balkanski, M., 2000."Elastic properties of crystals of single-walled Carbon Nanotubes", Solid State Commun., 114 pp. 395-399.

8. Qian,O. Wagner,G. J., 2003"Mechanics of Carbon Nanotubes", chapter 19 of hand book of CRC Press LLC.

9. Rafii-Tabar,[9]H., 2004."Computational modelling of thermo-mechanical and transport properties of Carbon Nanotubes", Physics Reports 390 -235-452

10. Saether, E. Frankland, S.J.V. Pipes,R.B., 2003." Nanostructured Composites: Effective Mechanical Poperty Determination of Nanotube Bundles " Comput. Sci. Technol. 